\title{
Arte, fenomenología y cuerpo femenino
}

\section{Artículo de investigación}

\section{Flor Emilce Cely Á.}

Universidad Nacional de Colombia ecelyf@unal.edu.co

Recibido: 2 de enero de 2019

Aprobado: 8 de marzo de 2020

Cómo citar este artículo: Cely, A. Flor Emilce (2021). Arte, fenomenología y cuerpo femenino. Calle 14: revista de investigación en el campo del arte 16(29). pp. 104-117.

https://doi.org/10.14483/21450706.17406

\section{(c) (1)}

https://creativecommons.org/licenses/by/4.0/deed.es 


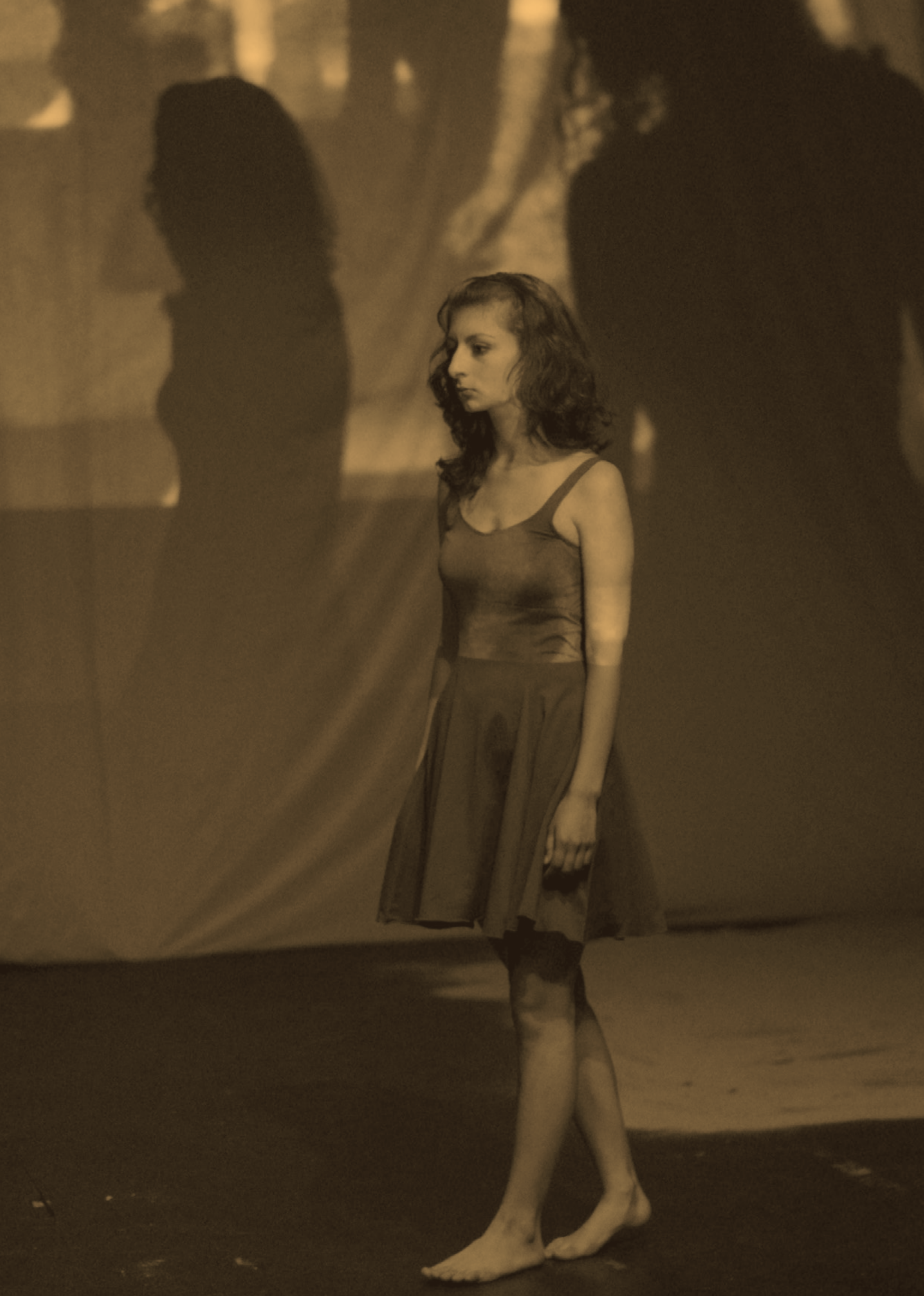




\title{
Resumen
}

Este artículo presenta dos maneras en que se relacionan el arte y la fenomenología. Por un lado, el aire de familia que hay entre la fenomenología y los logros del arte visual moderno en relación con una nueva manera de entender nuestro encuentro visual con el mundo y las ganancias a nivel experiencial que se derivan del mismo. $Y$, en segundo lugar, las lecciones que podemos extraer de los grandes artistas creadores y revolucionarios para conectarnos con nuestra experiencia, para volver a encontrarnos con nosotros mismos a partir de las nuevas maneras de ver que nos legaron. Se explorará en este sentido la relación arte/fenomenología con respecto al cuerpo, haciendo el contraste con la perspectiva científica del mismo, así como un análisis de la mirada artística y científica sobre el cuerpo de las mujeres a finales del siglo XIX y comienzos del XX.

Palabras clave

Arte; ciencia; cuerpo; fenomenología; mujeres

\section{Art, Phenomenology and the Female Body}

\begin{abstract}
This article presents two ways in which art and phenomenology are related. On the one hand, the family resemblance between phenomenology and the achievements of modern visual art in relation to a new way of understanding our visual encounter with the world and the gains at an experiential level that derive from it. And, secondly, the lessons that we can extract from the great creative and revolutionary artists to connect with our own experience, to find ourselves again with the new ways of seeing that they bequeathed us. In this sense, we will explore the art/phenomenology relationship with respect to the body, making a contrast with the scientific perspective on the subject, as well as an conducting an analysis of the artistic and scientific view of the body of women at the end of the 19th century and the beginning of the XXth.
\end{abstract}

\section{Keywords}

Art; science; body; phenomenology; women

\section{Art, phénoménologie et corps féminin}

\section{Résumé}

Cet article présente deux manières dont l'art et la phénoménologie sont liés. D'une part, la ressemblance familiale entre la phénoménologie et les réalisations de l'art visuel moderne en relation avec une nouvelle manière de comprendre notre rencontre visuelle avec le monde et les gains au niveau expérientiel qui en découlent. Et, deuxièmement, les leçons que l'on peut tirer des grands artistes créatifs et révolutionnaires pour se connecter avec notre propre expérience, pour se retrouver avec les nouvelles façons de voir qu'ils nous ont léguées. En ce sens, nous explorerons la relation art / phénoménologie par rapport au corps, en contraste avec la perspective scientifique sur le sujet, ainsi que la vision artistique et scientifique du corps de la femme à la fin du 19e siècle et début du XXe.

\section{Mots clés}

Art ; science ; corps ; phénoménologie ; femmes 


\section{Resumo}

Esse artigo apresenta duas maneiras pelas quais se relacionam a arte e a fenomenologia, Por um lado, o ar de família que há entre a fenomenologia e as conquistas da arte visual moderna em relação a uma nova maneira de entender nosso encontro visual com o mundo e os ganhos experienciais que se derivam do mesmo. E, em segundo lugar, as lições que podemos extrair dos grandes artistas criadores e revolucionários para conectarmos com nossa experiência, para encontramos com nós mesmos novamente a partir das novas maneiras de ver que nos legaram. Se explorará, neste sentido, a relação arte/fenomenologia em relação ao corpo, fazendo um contraste com a perspectiva científica do mesmo, assim como uma análise do olhar artístico e científico sobre o corpo das mulheres no final do século XIX e começo do século XX.

\section{Palavras chave}

Arte; ciência; corpo; fenomenologia; mulheres

Warmita ruraspa intirru kauachiku

\section{Maillallachiska}

Kai mailla kilkapi ninaku tiansi iskai parlu. Kauachii chasallata rurai kai iska, tapuchispa.

Intindichinaku, tukuikunata imami iacha ruragkuna munankuna kawanga. Nispa kunaura. Allilla tapuchispa maskaspa iachaikuspa sug mas iachakunaua kallariskakuna llugsichinga. Kanchama kai parlitukuna kai wata XIX tukuriura, iskai chunga wata kallariura.

\section{Rimangapa ministidukuna}

Ruraskakuna; iachaikui; nukanchi kikin; rurai iachaikuispa; warmikuna 
El propósito de este artículo es presentar algunas relaciones posibles entre la fenomenología y el arte, considerando diversas miradas del cuerpo. En primer lugar, se expondrá el 'aire de familia' que comparten sus respectivas actitudes hacia el mundo, particularmente en lo que tiene que ver con una nueva manera de entender nuestro encuentro visual con este, y las ganancias a nivel experiencial que se derivan del mismo. En un segundo momento, se explorará la relación arte / fenomenología con respecto al cuerpo, haciendo el contraste con la perspectiva científica del mismo. Esta indagación servirá para, finalmente, realizar un análisis sobre una manera muy particular de concebir el cuerpo de las mujeres en el arte, la fenomenología y la ciencia a finales del siglo XIX y comienzos del XX.

\section{Fenomenología y arte}

Son muy variadas y ricas las relaciones que se pueden establecer entre fenomenología y arte. Aquí solo haré mención a las interesantes referencias de MerleauPonty al arte pictórico. En particular, acerca de dos aspectos, en primer lugar, sobre las afinidades que se pueden encontrar entre la fenomenología y la pintura moderna. Y, en segundo lugar, las lecciones que podemos extraer de los artistas modernos que, con sus creaciones revolucionarias, nos mostraron cómo conectarnos con nuestra experiencia perceptual, cómo volvernos a encontrar con nosotros mismos a partir de las nuevas maneras de ver que nos legaron.

Respecto al primer punto se aprecia un hilo común entre los empeños de algunos artistas y la 'actitud fenomenológica'. Esta última fue propuesta por Husserl como 'reducción' (Husserl 1950), y consiste en hacer una descripción detallada de nuestra experiencia consciente, con el fin de captarla en toda su pureza, despojándola de toda la contaminación proveniente de la carga teórica, de preconcepciones del sentido común y de prejuicios, que están presentes cuando estamos en 'actitud natural'. A esto es a lo que se refiere Husserl cuando nos invita a 'dejar el mundo entre paréntesis', ese mundo sobrecognitivizado, que nos impide el encuentro íntimo con nuestro mundo experiencial más básico.

Merleau-Ponty va a retomar esta idea de la reducción propuesta por Husserl, enfatizando la necesidad de una descripción fiel de los fenómenos de los que tenemos experiencias vividas. Y, en particular, de los asociados con la percepción, pues habían sido dejado de lado u oscurecidos, tanto por parte de algunos sistemas filosóficos como por visiones científicas. De ahí que fue clara su intención de orientar la fenomenología como "...una filosofía para la cual el mundo siempre «está ahí», ya antes de la reflexión, como una presencia inajenable, y cuyo esfuerzo total estriba en volver a encontrar este contacto ingenuo con el mundo..." (Merleau-Ponty 1985, p. 7).

Pero esta tarea no es fácil. Y es para ello que MerleauPonty acude al arte, para mostrarnos, a partir del análisis de la experiencia del artista, particularmente del pintor, cómo es que recobramos ese contacto espontáneo con el mundo. Cómo es que, con Cézanne, por ejemplo, podemos aprender de nuevo a ver el mundo, a verlo verdaderamente ${ }^{1}$. En sus trabajos sobre arte, Merleau-Ponty se propuso mostrar que la actitud fenomenológica y una particular visión artística coinciden. “Para Merleau-Ponty, las pinturas de Cézanne hacen visible el movimiento hacia el 'ver fenomenológico', una suspensión de la actitud natural (que es asumida en la visión desde nuestras presunciones científicas y sesgadas en sentido práctico, sobre el ser espacial objetivo del mundo), para expresar la forma en que es constituida en nuestra visión". (Gosetti-Ferencei 2007, p. 197).

La pintura moderna es pues particularmente apta para ilustrar cómo podemos aprehender de manera diferente el mundo, en virtud del rechazo de la técnica de la perspectiva heredada desde el Renacimiento. Por ello será especialmente Cézanne quien llame la atención de Merleau-Ponty pues fue él quien logró captar en su pintura postimpresionista, no ya la noción de perspectiva, concepto científico, del que partieron los pintores clásicos, sino un espacio vivido: "Por su fidelidad a los fenómenos, los estudios sobre perspectiva de Cézanne descubrieron lo que la psicología sólo recientemente ha formulado: la perspectiva vivida, la de nuestra percepción, no es la perspectiva geométrica o fotográfica"2.

\footnotetext{
1 Hay que anotar aquí que este proceso de abstracción que está presente en la evolución de la pintura en Cézanne, “...puede encontrarse en una gran parte de las primeras vanguardias como el neoplasticismo, el simbolismo o el cubismo. Cada uno de estos movimientos, con métodos y aproximaciones diferentes, buscaba un retorno a un modo de ver que es primitivo, antes de la corrección que implica la perspectiva occidental". Agradezco esta observación hecha por un(a) evaluador(a) anónimo(a).

2 No se trata de que Merleau-Ponty considerara que las dos perspectivas fueran o implicaran lo mismo, solamente está indicando que nuestra perspectiva vivida es diferente, de un lado, de la perspectiva geométrica que se introdujo como un parámetro de medición objetiva en la ciencia y, de otro lado, de la perspectiva fotográfica que no se puede identificar con la geométrica, pero que también introdujo
} 
(Merleau-Ponty 2012, p. 32). Es más, para MerleauPonty, esta noción de perspectiva del Renacimiento "... que procede proyectando sobre un solo plano diferentes datos exteriores" tuvo una gran influencia cultural hasta tal punto que se constituyó en un modo de ver canónico en el adulto. De ahí que, para el autor francés, sean los niños los que comprendan de mejor manera el dibujo o la pintura moderna representados, por ejemplo, en la obra de Picasso (Merleau-Ponty, 1964).

Al igual que el arte moderno, Merleau-Ponty cuestionó entonces la concepción de la percepción que nos ofrecen las pinturas elaboradas a partir de la noción de la perspectiva, y que creemos es la natural, la más auténtica, cuando en realidad lo que nos brinda es una idea de percepción artificial.

El mundo de la percepción, es decir, aquel que nos revelan nuestros sentidos y la vida que hacemos, a primera vista parece el mejor que conocemos, ya que no se necesitan instrumentos ni cálculos para acceder a él, y, en apariencia, nos basta con abrir los ojos y dejarnos vivir para penetrarlo. Sin embargo, esto no es más que una falsa apariencia (...) en una gran medida es ignorado por nosotros, mientras permanecemos en la actitud práctica o utilitaria (...) hizo falta mucho tiempo, esfuerzos y cultura para ponerlo al desnudo y que uno de los méritos del arte y el pensamiento modernos (...) es hacernos redescubrir este mundo donde vivimos pero que siempre estamos tentados de olvidar. (Merleau-Ponty 2006, p. 9).

En este sentido, encuentra en Cézanne una maravillosa capacidad de distorsión que "...saca a la luz los tejidos de la estructura perceptual, en lugar de presentar al mundo en su supuesto realismo natural. Si se produce una distorsión en la pintura de Cézanne, debe entenderse como un rechazo de la perspectiva óptica (y de las técnicas de representación dimensional desde el Renacimiento) y un rechazo de la verosimilitud artificial" (Gosetti-Ferencei 2007, pp. 158-159).

Ahora bien, hay un sentido en que es cierto que los objetos se nos presentan a la mirada desde una única perspectiva. Se trata de aquella a partir de la cual los objetos se nos ofrecen, dejándonos ver uno solo de sus perfiles, quedando ocultos sus aspectos recesivos. No obstante, percibimos las cosas como un todo unificado, captamos las cosas como existentes más allá del conocimiento actual:

Toda experiencia tiene su horizonte experiencial; cada una tiene su núcleo de conocimiento real y determinado, tiene su contenido de determinaciones inmediatamente dadas por sí mismas, pero, por encima de este núcleo de un ser-así determinado, de lo que propiamente se da como "ello mismo ahí", posee ella su horizonte. Esto quiere decir: toda experiencia remite a la posibilidad y, a partir del yo, a una re-posibilidad (Husserl 1980, p. 33).

Captamos un objeto perceptivamente desde una perspectiva particular que nos aporta un solo perfil, pero los otros perfiles hacen parte de un horizonte de posibilidades que se abre gracias a la invitación a interactuar que recibimos del objeto, y de la certeza que tenemos de que la percepción que los otros tienen -o han tenido- del mismo objeto, complementan esos perfiles para que se nos presente como un todo. Por ejemplo, el hecho de no percibir o tocar la taza de café en su totalidad, no nos lleva a desconfiar que le falte el pedazo que no vemos o tocamos y a temer que nuestros dedos se sumerjan en el líquido - como en una escena de cine ficción. La taza de café se me presenta a la vista y al tacto con unos perfiles determinados, que ocultan otros (escorzos latentes), pero su presencia no se agota allí, pues también se me presenta como un 'horizonte de expectativas'.

La fenomenología nos invita pues a comprender que el mundo de la percepción es un mundo rico que se constituye gracias a la conjunción dinámica de los sentidos, los afectos y las experiencias sensibles de los otros. Y, en esta medida, nos invita a volver a aprender a percibir, pues nosotros en nuestra cotidianidad, así como el científico en lo suyo, estamos atrapados en un punto de vista singular que reduce el objeto a una mirada sesgada, orientada por pensamientos y creencias previas, prejuicios o teorías.

Como es sabido, esta concepción de la percepción en la fenomenología nace de un cuestionamiento del dualismo cartesiano mente/cuerpo y de la sospecha de que la noción de espacio vivido no coincide con la noción de espacio físico, esto es, el espacio geométrico definido según las reglas de la ciencia. No habitamos ese espacio físico, la nuestra es una espacialidad de situación.

El espacio ya no es aquel del que habla la Dióptrica, una red de relaciones entre objetos, tal como 
la vería un tercer testigo de mi visión, o un geómetra que la reconstruye y la sobrevuela; es un espacio contado a partir de mí como punto o grado cero de la espacialidad. No veo el espacio según su envoltura exterior, lo veo desde dentro, estoy englobado en él. Al cabo, el mundo está alrededor de mí, no delante de mí. (Merleau-Ponty 2013, p. 46).

De ahí la sintonía encontrada por Merleau-Ponty en la pintura de Cézanne, pues este logró captar un espacio "completamente denso y lleno" (Prendeville 1999, p. 379). Es un ejemplo supremo de que "la pintura da existencia visible a lo que la visión profana cree invisible, hace que no tengamos necesidad de un «sentido muscular» para tener la voluminosidad del mundo" (Merleau-Ponty 2013, p. 27)33. Así, Merleau-Ponty ubica en este tipo de pintura el origen, el nacimiento del significado: la pintura se expresa, 'habla' (una clase de habla, una clase de inscripción de la visibilidad en el mundo) a través del gesto del sujeto corporizado-vivido (Gosetti-Ferencei, 2007).

En síntesis, tenemos de un lado la 'percepción perdida' en el cálculo y la exactitud científicas (expresadas en la noción de perspectiva del Renacimiento). De este lado está el mundo realista; un mundo reflexivo, visto objetivamente. Es el mundo del científico y del artista que hace mediciones, que construye un modelo de la realidad y después se queda con él y deshecha el original. Esto le reclama Merleau-Ponty al pintor premoderno: "Cuando el pintor guiña los ojos, destruye la organización en profundidad del campo y, con ella, los contrastes precisos de la iluminación; no hay ya cosas determinadas con sus colores propios." (Merleau-Ponty 1985, p. 322).

Y, de otro lado, está la 'percepción recobrada' que, gracias a la actitud fenomenológica, nos revela el mundo; es el mundo de la experiencia que nos es dado gracias a nuestra corporeidad; un mundo de objetos que nos ofrecen posibilidades de acción, y de desdoblamiento de la perspectiva. Y que en la pintura moderna se expresa en su captación de la profundidad. Noción que habíamos perdido con el el énfasis en la perspectiva y que expresa el acople especialmente agudo entre percepción y existencia (Morris 2004, p. 2). Volveremos más adelante sobre esta noción.

3 Ver, por ejemplo, las obras de Cézanne, Naturaleza muerta con escayola, 1894 o Bibemus Quarry de 1900.
Uno de los aportes más valiosos de la fenomenología de la percepción merleaupontiana es que con el 'ver fenomenológico' como experiencia vivida, nos mostró que es posible conectarnos de formas renovadas con todas las posibilidades de nuestro propio cuerpo. Y esto no de una manera solipsista, porque esa renovación de experiencias corporizadas no es algo distinto a la renovación del mundo que capto con ellas, y a la reinvención de las relaciones con otras subjetividades corporizadas. Veamos.

\section{Cuerpo en el arte, en la fenomenología y en la ciencia}

¿Con qué estamos más familiarizados? Con nuestras 'mentes', dirán algunos. Pero en realidad constatamos cotidianamente una extraña familiaridad con nuestras habitualidades corporizadas. Extraña puesto que, de un lado, nada más familiar que nuestra conciencia corporizada prerreflexiva, pues es gracias a diversas modalidades de expresión de nuestra corporalidad dinámica y afectiva que nos conectamos con ese mundo de todos los días. Si, por ejemplo, perdiéramos ese 'sentido de familiaridad', esas rutinas mínimas de todos los días se nos harían enormemente difíciles, la existencia se tornaría perturbada, como sucede con el esquizofrénico (Blankenburg, 2001).

Pero, de otro lado, el cuerpo como un entrelazamiento de visión, tacto y cinestesias, ese cuerpo operante y actual del que nos habla Merleau-Ponty en El ojo y el espíritu, nos es indiferente, pues funciona tácitamente, como 'en automático'. De lo contrario, nuestros movimientos, nuestro andar por el mundo no sería fluido (como les sucede por ejemplo a las personas que pierden su sentido de la propiocepción y deben acompañar cada movimiento con la mirada). Se puede hablar aquí como de una transparencia del cuerpo ${ }^{4}$ : hasta cierto punto debo desentenderme de mi cuerpo para que funcione, para que fluya en sus tareas cotidianas de hacerme presente en el mundo, de darme un mundo.

Y entonces adoptar la actitud fenomenológica implica aquí una nueva manera de 'ver el mundo' que no es ya una actitud pasiva ante el mismo, sino una captación corporizada, dinámica de lo que tenemos delante. Nada más natural entonces que considerar al cuerpo como el

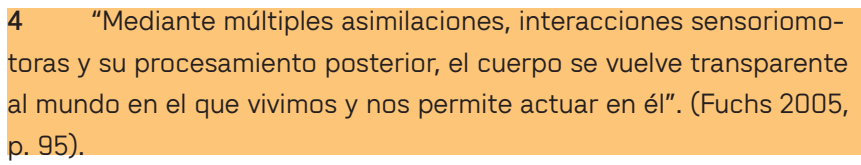


punto cero de orientación (Husserl 2005), el eje o punto de referencia imprescindible de toda visión verdadera del mundo.

Retomemos el horizonte lleno de expectativas que mencionábamos en la sección pasada, que hace posible percibir los objetos como un todo unificado y no parte a parte. Ese horizonte es explorado por una corporalidad que no simplemente capta las sensaciones para que sean después unificadas en un concepto (como en Kant) o procesadas representacionalmente (como en el cognitivismo). Si necesitamos suponer una síntesis, es el cuerpo el que la lleva a cabo. Es mi cuerpo el que me pone en contacto con el mundo, me da un mundo ya significativo: tomo la taza de café porque es agarrable, porque hay algo en ella — tanto en su forma, como en su olor, como en los afectos asociados- que incita mi ímpetu por tomar el café bien caliente y me arranca unos movimientos que finalmente terminan esa en esa acción, que pareciera milimétricamente planeada, pero que en realidad es más automática de lo que parece. “Todo lo que veo está por principio a mi alcance, por lo menos al alcance de mi mirada, consignado sobre el mapa del «yo puedo». Cada uno de estos dos mapas es completo. El mundo visible y el de mis proyectos motores son partes totales del mismo Ser" (Merleau-Ponty 2013, p. 21-22).

Hablamos entonces de la percepción como una 'captación', como una aprehensión directa, automática, de aquello que nos interesa, de lo que nos incita, y nos mueve a emprender un curso de acción en el mundo perceptivo. Y no como un proceso en el que, primero vemos, captando elementos inconexos, como una tarea parcial que tendría que ser siempre complementada por la formación de representaciones, por un procedimiento inferencial, o por una explicación que incluya las causas. Así, la fenomenología está más del lado del arte que de la ciencia. Paradójicamente esta última, al proponerse mantenerse fiel a un supuesto realismo, termina estudiando un mundo artificial, perfilado con la linealidad y atomicidad de la mirada causalista, y a un cuerpo que termina reduciendo a mero cuerpo-objeto, gobernado por las leyes de la mecánica, dejando de lado su dimensión existencial como un cuerpo vivido. En cambio, la fenomenología nos invita a conectarnos con lo real del mundo y de nuestro propio cuerpo, esto es, unidad, presencia y plenitud, expresadas tan maravillosamente en el arte moderno, estilo Cézanne:

En la percepción primaria, estas distinciones entre tacto y vista no existen. La ciencia del cuerpo humano es la que nos acaba enseñando a diferenciar nuestros sentidos. El objeto percibido no es rescatado o construido a partir de los datos proporcionados por los sentidos, sino que se nos muestra de entrada como el centro de donde proceden esos datos. Vemos la profundidad, la textura, la suavidad, la dureza de los objetos -para Cézanne, incluso vemos su olor. Si el pintor ha de expresar el mundo, la disposición de los colores deberá llevar en sí ese Todo indivisible; de lo contrario, su pintura sólo será una alusión a las cosas y no las reflejará en esa unidad imperiosa, esa presencia, esa plenitud ineludible en la que radica nuestra definición de lo real. (Merleau-Ponty 2012, p. 43).

Lo que la fenomenología le reclama a la ciencia es, por un lado, la exclusión de su campo de estudio de la esfera experiencial, de la perspectiva de primera persona. Es decir, la ciencia tiene mucho que aprender de esos 'modos de ver' distintos, captados en la descripción fenomenológica y expresados en el arte. Ha de aceptar la invitación a aprender a ver el mundo, a los otros y la propia subjetividad corporizada, con toda su riqueza experiencial, esto es, con sus rasgos salientes dinámicos, afectivos y situados. Merleau-Ponty nunca renunció a aspirar a que la ciencia se enmendara de esta manera:

Es preciso que el pensamiento de ciencia - pensamiento de sobrevuelo, pensamiento del objeto en general- se resitúe en un «hay» previo, en el sitio, sobre el suelo del mundo sensible y del mundo abierto tal como son en nuestra vida, para nuestro cuerpo, no ese cuerpo posible del que se puede sostener con facilidad que es una máquina de información, sino este cuerpo actual que llamo mío, el centinela que se mantiene silenciosamente por debajo de mis palabras y de mis actos. (Merleau-Ponty 2013, p. 19).

Algo que hemos comenzado a presenciar gracias a los contactos interdisciplinarios entre fenomenología y ciencias cognitivas, y que ha permitido que los científicos se interroguen por las maneras de integrar el aspecto experiencial en sus investigaciones. (cf. por ejemplo, Varela \& Shear 1999).

Pero no fue solamente la ciencia la que intentó reducir el mundo a una mirada objetivista. En el Renacimiento los pintores, con su noción de perspectiva, asimilaron la mirada objetivista que se basaba en una concepción científica de 'espacio objetivo' y geometría euclidiana. 
Esta mirada se basaba en una 'fórmula tripartita': objeto, sujeto y espacio. El objeto como lo que se experimenta, el sujeto con su perspectiva trascendente desde la cual se tiene la experiencia, y el espacio como un medio continuo a través del cual se produce la experiencia. Ahora bien, es interesante constatar, como lo señala Rosen (2008), que estas tres categorías consideradas mutuamente excluyentes en el pensamiento clásico, fueron superadas por una relación de mutua inclusión, expresada en la dimensión primitiva de la profundidad propuesta por Merleau-Ponty, en la botella de Klein de la topología y en el espacio visual de Cézanne. De manera tal que, en la primera mitad del siglo XX, fue más que una feliz coincidencia la confluencia entre fenomenología, modelos matemáticos y arte moderno en torno al cuestionamiento de una concepción de espacio y tiempo absolutos, así como de la geometría euclidiana y de la técnica de perspectiva.

Además de esa mirada linear causalista, la ciencia se caracterizó por dejar de lado el hecho de que nuestros cuerpos son cuerpos vividos, que no pueden reducirse a cuerpos físicos percibidos o investigados desde la perspectiva de tercera persona. Se trata de cuerpos que experimentan sensaciones y que tienen experiencias del mundo desde su particular perspectiva o punto de vista. Pero también se trata de subjetividades corporizadas que de manera ineludible se co-constituyen en los encuentros interafectivos con otras subjetividades corporizadas, en el marco de una situación social y cultural que le pone un sello específico a esa co-constitución, sedimentando en cada uno de nosotros unas formas particulares de ser.

Para terminar, propongo en la última sección un ejemplo específico que permite entender el lazo especial que hay entre la 'mirada fenomenológica' y la 'mirada artística', en contraste con la 'mirada científica', que termina siendo objetualizadora. Se trata de recorrer parte de la historia de la mirada de los cuerpos femeninos en una época específica (finales del siglo XIX y comienzos del XX), y en un lugar específico, Viena, centro del desarrollo científico, artístico y filosófico de esas décadas. El objetivo de esta reflexión es mostrar cómo el despliegue de maneras distintas de ver — tal como lo desarrollamos aquí respecto a la fenomenología, el arte y la ciencia - está imbuido por preconcepciones e imaginaciones sociales que, en últimas, determinan las realidades de los cuerpos. Pretende analizar a partir de la fenomenología la manera específica en que la mirada científica, de un lado, y la mirada artística, de otro, instauraron dos modos distintos de percibir los cuerpos femeninos, a partir de trasfondos disímiles y con consecuencias distintas para la subjetividad de cada mujer y para la construcción social del cuerpo de las mujeres.

\section{El cuerpo de las mujeres. Ciencia y arte, primera mitad del siglo $\mathrm{XX}$}

Esta época se caracterizó porque nuevos horizontes de mirada se desplegaron gracias a varios hechos. En primer lugar, estuvo el boom de la fotografía desde el siglo XIX que, además de sus contribuciones artísticas, sirvió también como un instrumento de investigación científica, y dio también rienda suelta a nuevas formas de satisfacción voyeurística. En segundo lugar, con la apertura de los grandes hospitales psiquiátricos (como el Steinhof en 1907), se abrieron nuevas perspectivas para el voyeur. A ellos se podía ir para contemplar el 'espectáculo' que algunos enfermos exhibían con sus cuerpos desfigurados, retorcidos. Se trataba de la deformidad del cuerpo como espectáculo, pero también de las expresiones gestuales y de cuerpo entero de las llamadas histéricas. Estas mujeres fueron objeto de miradas contemplativas tanto por parte de profesionales de la salud, como Charcot y sus jóvenes médicos en formación, como también de artistas médicos (como Paul Richer) encargados de dibujarlas, y de fotógrafos como Bourneville \& Regnard quienes organizaron, junto con Charcot, un registro fotográfico de ellas justo en el momento en que padecían ataques que incluían convulsiones, contracción de miembros y un sinfín de movimientos y poses que fueron clasificados por el propio Charcot como 'actitudes pasionales' (cf. Iconographie Photographique de la Salpêtrière). En síntesis, el cuerpo se convirtió en esta época en un objeto público (Erbguth 2010, p. 56).

El punto es que todas estas prácticas fueron creando una atmósfera que al mismo tiempo que confirmaba, ayudaba a extender la idea de la mujer como un ser pasional, sexualizado, desbordado por las manifestaciones corporales de su enfermedad. Tanto que inclusive llevó a las mismas pacientes a la identificación con sus cuerpos histéricos. Cuerpos observados con inquietud científica, pero también con la curiosidad del médico-pintor, del médico-fotógrafo y, por supuesto, también del artista.

Como se sabe, Freud jugó un papel importante en el tratamiento de estas pacientes (Freud \& Breuer 1976) y su aporte es recordado en parte porque tomó distancia de la perspectiva científica de Charcot centrada 
en la mirada, y le abrió un espacio a la escucha de los relatos de estas mujeres sobre los problemas que padecían, muchas veces a causa de acosos o abusos en su infancia o juventud. Y con la apertura de este espacio de escucha fue creando el método psicoanalítico de la asociación libre, y al mismo tiempo fue elaborando su teoría psicoanalítica de la psique humana. Ahora bien, a pesar de la importancia de estos avances, hay dos problemas importantes con la teorización y tratamiento freudianos. En primer lugar, la cancelación de la mirada en el dispositivo analítico clásico: el analista está ubicado detrás del diván donde el paciente está recostado haciendo su asociación libre. Si bien es cierto que no estaban justificados los procedimientos contemplativos (para algunos voyeristas) y las presentaciones teatrales que organizaban Charcot y sus colegas con la demostración pública de 'síntomas histéricos', fue erróneo proscribir de la indagación del médico una 'mirada experta' acerca de la corporeidad de sus pacientes pues, a través del cuerpo, como vehículo del mundo, podemos apreciar también la expresión de rasgos o eventos significativos en la vida de una persona, que se escapan a la formación de representaciones y a la puesta en palabras (Merleau-Ponty 1985). En la psiquiatría contemporánea de orientación fenomenológica se ha rescatado este aspecto dándole un lugar importante a la captación de aspectos corporizados (gestos, expresiones faciales, posturas corporales, estilos de movimiento, etc.) como expresión de profundas anormalidades que, por su misma naturaleza, escapan a la posibilidad de ser verbalizadas ${ }^{5}$.

Y, en segundo lugar, hay problemas importantes con la actitud verificacionista de Freud (Popper 1985), que hizo que cualquier evento relatado en el discurso de sus pacientes, desde los más significativos hasta los más triviales, fueran encuadrados dentro de su teoría del origen sexual reprimido de los síntomas histéricos. Solo hace falta revisar los casos que publicó con su colega Breuer y en particular el famoso y controvertido caso Dora. Este caso es una muestra de la manera en que Freud buscó siempre la forma de verificar su hipótesis de la etiología sexual de las neurosis, llegando con ello a negarle credibilidad a las palabras de esta joven, quien siempre manifestó repulsión por los continuos acosos a los que era sometida por un hombre mayor amigo de la familia. Freud llegó a sostener inclusive que, en realidad, los síntomas psicosomáticos de Dora manifestaban un

5 Cf. por ejemplo, (Parnas \& Zahavi, 2002) y para una revisión de la propuesta de terapias en movimiento, derivada de esta concepción, (Röhricht et al. 2014) deseo sexual inconsciente por este hombre, por su propio padre, o bien por el mismísimo Freud. (Kandel, 2013)

Ahora bien, mientras tanto en esa misma Viena, por la misma época, en el taller de los artistas que iban fraguando el movimiento expresionista, se dibujaban y pintaban artísticamente los cuerpos de mujeres y hombres desnudos, atravesados por el pathos de la pasión y el sufrimiento, tal como lo expresan las obras de Schiele ${ }^{6}$. Y fue inevitable, en esas coordenadas espacio-temporales, que se entablara una relación entre este movimiento artístico y el estudio y tratamiento científico de enfermedades mentales. Volvamos a Charcot. Recordemos que en 1876-1880 publicó su Iconografía Fotográfica, dedicada a reunir fotografías de gestos y expresiones dramáticas de la histeria, una histeria con sesgo de género y erotizada (Bernheimer \& Kahane, 1985). Pero lo que es menos conocido es que ocho años después lanzó su Nueva Iconografía (Nouvelle Iconographie de la Salpêtrière: Clinique des Maladies du Système Nerveux, 1888-1918 - NIS), que incluía fotografías con una orientación distinta: imágenes de las deformidades del cuerpo a causa de patologías neurológicas, tanto de mujeres como de hombres. Se trató de una publicación seriada en la que los artistas modernos buscaron nuevas iconografías del cuerpo.

Lo que tal vez vemos en este esfuerzo sostenido de destacar la "invasión" de la patología en el arte y del arte en la imaginería de la patología, es la expectativa de que la revista NIS atraería no solo a los médicos, sino a los artistas. Me gustaría argumentar inclusive que, al privilegiar la imagen, la revista se promocionó como un libro de referencia para artistas modernos que buscaban nuevas iconografías del cuerpo. (Blackshaw 2007, p. 388)

Schiele, por supuesto, estuvo familiarizado con las ideas de la representación de la patología en los retratos. Estuvo trabajando en Viena, la ciudad que fue el centro de los avances psiquiátricos en Europa, fue muy cercano a un artista que pintaba pacientes en el hospital (Osen) y a un médico que estuvo muy interesado en la expresión patológica (Kronfeld). De ahí que se interpreten algunos de sus autorretratos — como hace Gemma Blackshawno ya como la expresión de sus conflictos y demonios internos, sino como creados a partir de la influencia de esta serie de registros fotográficos. Así pues, tenemos en Schiele el 'compromiso' básico del pintor con el mundo que tiene ahí en frente y que lo arrojó a plasmar

6 Ver, por ejemplo, su obra Umarmung, 1912 
cuerpos masculinos y femeninos de esa particular manera. Recordemos que se trata de la herencia de una forma de ser de la pintura que se inició con Cézanne y que hizo patente, como ninguna filosofía hasta entonces, cómo la visión se hacía gesto. “Cézanne nos hace ver lo que está sucediendo siempre en la visión pero que, dado que se dilata más allá de los focos cotidianos de la actitud natural, es algo de lo cual no nos damos cuenta. La pintura, se podría decir, es la expresión gestual de la visión". (Gosetti-Ferencei 2007, p. 200).

En síntesis, el cuerpo de las mujeres, visto a través de los lentes del extendido discurso europeo como un cuerpo sexualizado e histérico, encontró nuevas maneras de ser visto, de verse y expresarse en la re-visión del cuerpo llevado a cabo por los expresionistas vieneses. Una vanguardia que, como nos lo recuerda Timpano "fue impulsada por el deseo de mostrar a la sociedad contemporánea el 'nuevo' cuerpo como filtrado a través de una sensibilidad moderna". (Timpano 2017, p. 45).

Tuvimos entonces en la Viena de esa época la confluencia de formas de ver, de perspectivas muy particulares sobre el cuerpo y, en particular sobre el cuerpo femenino. Por un lado, y paradójicamente, la mirada psicoanalítica freudiana, por más que pretendió marcar una ruptura con las miradas científicas de la época sobre la histeria, no logró escapar a esa influencia pues, en el trasfondo de su teoría y de su práctica psicoterapéutica reinó una preconcepción del cuerpo femenino imbuida por las nociones científicas corrientes de cuerpos sufrientes a causa de un deseo sexual reprimido, y que no le permitió llegar a una idea amplia de la sexualidad femenina, al alinearse con una concepción estrecha de la misma como algo misterioso. De otro lado, en los talleres de los artistas que estaban marcando la transición hacia nuevas formas de hacer arte, se dio el espacio para la expresión de cuerpos que se sentían libres para manifestarse de maneras naturales, espontáneas ${ }^{7}$. Libres en su expresión, pero también liberadas del peso de esa mirada científica de la época que pretendió circunscribir su deseo sexual al registro de lo anómalo, de lo raro que ha de ser patologizado, recluido y registrado cuidadosamente, por una comunidad científica que no estuvo libre de la sospecha de que, como voyeurs, también disfrutaban del espectáculo.

Desde el comienzo de este escrito nos hemos empeñado en hacer patente la importancia de la labor

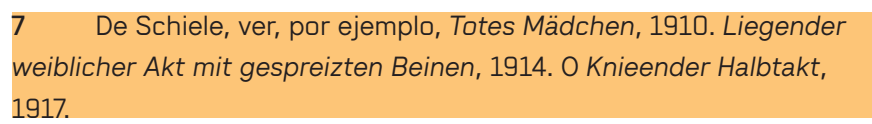

fenomenológica de recobrar el mundo perdido de la percepción; hemos hecho eco del llamado en el que coinciden Merleau-Ponty y el modernismo inaugurado por Cézanne, de aprender a ver verdaderamente. Ahora bien, de otro lado, cuestionamos los intentos de los médicos de finales del XIX y comienzos del XX en relación con agudizar su mirada y acudir a expertos en otras 'formas de ver' como los fotógrafos y los pintores para registrar los gestos y posturas de las pacientes histéricas. No obstante, es lícito defender que esos ensayos científicos de comienzos del siglo XX tuvieron un sentido, pues se los puede interpretar como intentos importantes de entender de forma integral a sus pacientes ${ }^{8}$.

Charcot sostenía que la fotografía era más precisa que la observación médica, ya que registraba la patología bajo escrutinio, lo que permitía al médico estudiarla repetidamente. La imagen del cuerpo del paciente fue, por lo tanto, fundamental para el diagnóstico y tratamiento de la enfermedad neurológica. Las fotografías proporcionaron a los médicos un vocabulario visual para definir la neuropatología, permitiendo que diversas categorías de enfermedades fueran construidas y difundidas entre las comunidades médicas. (Blackshaw 2007, p. 386)

Recordemos que la utilidad actual de adoptar una perspectiva fenomenológica, en terrenos de intervención como en la psicología clínica o en la psiquiatría, reside en su propuesta de complementar la escucha con una captación visual de rasgos perturbados de la experiencia corporizada y su correspondiente tratamiento a partir del movimiento. Esta fue una de las grandes lecciones de Merleau-Ponty luego de su detallado estudio del caso Schneider: una comprensión correcta y una vía efectiva de tratamiento de sus síntomas se hubiera dado si se hubiera tenido en cuenta que sus afecciones visuales, táctiles, cognitivas y motoras conformaban un todo que no se puede desglosar con el fin de encontrar antecedentes causales. (Merleau-Ponty, 1985, sección "La espacialidad del propio cuerpo y la motricidad").

De manera tal que tomar en serio la corporización, esto es, nuestra naturaleza fundamentalmente dinámica, afectiva, intersubjetiva y situada, nos tiene que llevar a renovar los diversos modos del autoconocimiento, pues no es cierto que éste tenga que ver solamente

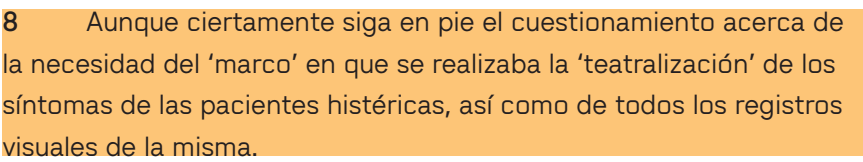


con poner en palabras, o explorar contenidos proposicionales conscientes o inconscientes. Tiene que ver con conectarnos con ese mundo de experiencia básico, con encontrar el cuerpo que somos y al que no le dirigimos la atención por estar interesados en 'nuestras mentes'.

\section{Conclusión}

Con el psicoanálisis freudiano se fundó y arraigó la idea de que con discurso podemos cambiar los pensamientos, las decisiones, las acciones. De que con la palabra hablada y la escucha del psicoanalista, se elimina el síntoma y el conflicto y se produce la cura. De ahí que el paciente le da la espalda al terapeuta, de ahí que en ese encuadre psicoterapéutico se le sacara el cuerpo al cuerpo. Propusimos acá hacer un recorrido con el cual se pudiera apreciar por qué no debemos despreciar la visión, lo que la mirada -experta, del clínico fenomenólogo, o la sensible, del artista- nos ofrece. Desde que nacemos somos seres en el movimiento y la visión (y la escucha, el tacto, y demás). Recortar, impedir la mirada hacia el otro y hacia sí mismo es impedir un acceso con posibilidades maravillosas, es obligar a 'palabrear' toda nuestra experiencia, a centrarnos en el discurso. Lo que debemos hacer ahora nosotros, sujetos herederos del modernismo, es aprender las lecciones que nos dejó este movimiento artístico, y aprender verdaderamente a ver, tanto ver el mundo, como a nosotros mismos. Dejarnos llevar por el arrebato de Cézanne que se abalanzaba sobre un lienzo que demoraba mucho en empezar a pintar y que no abandonaba hasta no estar seguro de haberlo llenado con espacios densos, con volúmenes, formas y movimientos tan vivos como los de la experiencia. Entender además que nuestra existencia se encuentra atravesada por la mirada de los otros, una mirada que puede estar cargada por prejuicios y limitaciones que terminamos asimilando como propias, como parte de nuestra propia identidad (como fue el caso con las 'mujeres histéricas').

En esto, como esperé haber mostrado, nos da una gran lección el arte moderno, pues nos enseña cómo romper con la experiencia ordinaria del "ver" para aprender a ver de nuevo. Esto es, a ver de maneras diferentes que transgredan nuestra experiencia habitual, que provoquen un encuentro absolutamente visual que permita el surgimiento del significado dentro de un 'éxtasis silente de la visión' (Gosetti-Ferencei 2007).

\section{Referencias}

Bernheimer, C., \& Kahane, C. (1985). In Dora's Case

Freud - Hysteria - Feminism. New York: Columbia University Press.

Blackshaw, G. (2007). "The Pathological Body: Modernist Strategising in Egon Schiele's SelfPortraiture". Oxford Art Journal 30 (3), 379-401.

Blankenburg, W. (2001). "First Steps toward a Psychopathology of "Common Sense"". Philosophy, Psychiatry, \& Psychology, 8 (4), pp. 303-315.

Erbguth, F. (2010). “Egon Schiele and Dystonia". En J. E. Bogousslavsky, Neurological Disorders in Famous Artists - Part 3. Frontiers of Neurology and Neuroscience Vol. 27 (págs. 46-60). Basel: Karger.

Freud, S., \& Breuer, J. (1976). Estudios sobre la histeria (1893-95). En: Obras Completas, vol. II. Buenos Aires: Amorrortu.

Fuchs, T. (2005). “Corporealized and Disembodied Minds. A Phenomenological View of the Body in Melancholia and Schizophrenia". Philosophy, Psychiatry \& Psychology, 12 (2), 95-107.

Gosetti-Ferencei, J. (2007). The Ecstatic Quotidian. Phenomenological Sightings in Modern Art and Literature. Pennsylvania : Pennsylvania State University Press.

Husserl, E. (1950). Ideas relativas a una fenomenología pura y a una filosofía fenomenológica. México: FCE.

. (1980). Experiencia y juicio: investigaciones

acerca de la genealogía de la lógica. México: UNAM, Instituto de Investigaciones Filosóficas.

(2005). Ideas relativas a una

fenomenología pura y a una filosofía fenomenológica. Libro segundo. Investigaciones fenomenológicas sobre la constitución. México: Fondo de Cultura Económica.

Kandel, E. (2013). La era del inconsciente. La explicación del inconsciente en el arte, la mente y el cerebro.

Madrid: Paidós.

Merleau-Ponty, M. (1964). The Primacy of Perception.

Northwestern : Northwestern University Press. 
(1985). Fenomenología de la percepción.

Barcelona: Península.

- (2006). El mundo de la percepción. Siete conferencias. México: Fondo de Cultura Económica. (2012). La duda de Cézanne. Madrid:

Casimiro.

. (2013). El ojo y el espíritu. Madrid: Trotta.

Morris, D. (2004). The sense of space. New York: State University of New York.

Parnas, J., \& Zahavi, D. (2002). "The role of phenomenology in psychiatric diagnosis and classification". En M. Maj, \& et al., Psychiatric Diagnosis and Classification (pp. 137-162). Chichester: John Wiley \& Sons.

Popper, K. (1985). Post Scriptum a la lógica de la investigación científica. Vol. I. Realismo y el objetivo de la ciencia. Buenos Aires: Paidós.
Prendeville, B. (1999). "Merleau-Ponty, Realism and Painting: psychophysical space and the space of exchange". Art History, 22 (3), 364-388.

Röhricht, F. et al. (2014). “Embodied cognition and body psychotherapy: The construction of new therapeutic environments". Sensoria: A Journal of Mind, Brain \& Culture 10 (1), 11-20.

Rosen, S. (2008). “Quantum Gravity and Phenomenological Philosophy". Foundations of Physics 38 (6), 556-582.

Timpano, N. (2017). Constructing the Viennese Modern Body: Art, Hysteria, and the Puppet. New York: Routledge.

Varela, F., \& Shear, J. (1999). The view from within:

First-person approaches to the study of consciousness. London: Imprint Academic. 